Mappemonde

Revue trimestrielle sur l'image géographique et les formes du territoire

$125 \mid 2019$

Varia

\title{
Conjuguer géomatique et design : Châto-Carto, cartes sensibles de Wangenbourg-Engenthal
}

\section{Anne-Christine Bronner}

\section{OpenEdition}

\section{Journals}

Édition électronique

URL : http://journals.openedition.org/mappemonde/804

DOI : $10.4000 /$ mappemonde.804

ISSN : 1769-7298

\section{Éditeur}

UMR ESPACE

\section{Référence électronique}

Anne-Christine Bronner, «Conjuguer géomatique et design : Châto-Carto, cartes sensibles de Wangenbourg-Engenthal », Mappemonde [En ligne], 125 | 2019, mis en ligne le 01 janvier 2019, consulté le 01 mars 2021. URL : http://journals.openedition.org/mappemonde/804 ; DOI : https:// doi.org/10.4000/mappemonde.804

Ce document a été généré automatiquement le 1 mars 2021.

\section{cc) (†) (อ)}

La revue Mappemonde est mise à disposition selon les termes de la Licence Creative Commons Attribution - Pas d'Utilisation Commerciale - Partage dans les Mêmes Conditions 4.0 International. 


\title{
Conjuguer géomatique et design : Châto-Carto, cartes sensibles de Wangenbourg-Engenthal
}

\author{
Anne-Christine Bronner
}

1 L'exposition Cartologies ${ }^{1}$ du colloque Temps, Art \& Cartographie - La sémiologie dans tous les sens a été initiée dans l'objectif de faire travailler des groupes d'étudiants autour du lien entre Art et Cartographie. C'est ainsi que le master géomatique OTG (Observation de la Terre et géomatique de la Faculté de géographie et d'aménagement de Strasbourg) et l'InSituLAB (DSAA, diplôme supérieur des arts appliqués du lycée Le Corbusier d'Illkirch-Graffenstaden) se sont associés autour d'un projet commun qui s'est concrétisé sous la forme d'un workshop de trois jours (24-26 février 2016) avec 38 étudiants (14 géographes + 24 designers). Il était encadré par Nicolas Couturier, Bruno Lavelle, Carmen Princelle et Danielle Martin (InSituLAB), Anne Puissant et AnneChristine Bronner (Faculté de géographie et d'aménagement).

2 Il s'agissait d'un premier jalon d'un projet plus large que menait l'InSituLAB ${ }^{2}$, qui avait pour but de prendre connaissance du territoire de la commune de WangenbourgEngenthal. L'enjeu était de créer, par groupe, un jeu de cartes sensibles du territoire de la commune, cartes qui allaient être exposées dans le cadre de l'exposition associée au colloque, puis sur place (figure 1). 
Figure 1. Quelques cartes du projet Châto-Carto

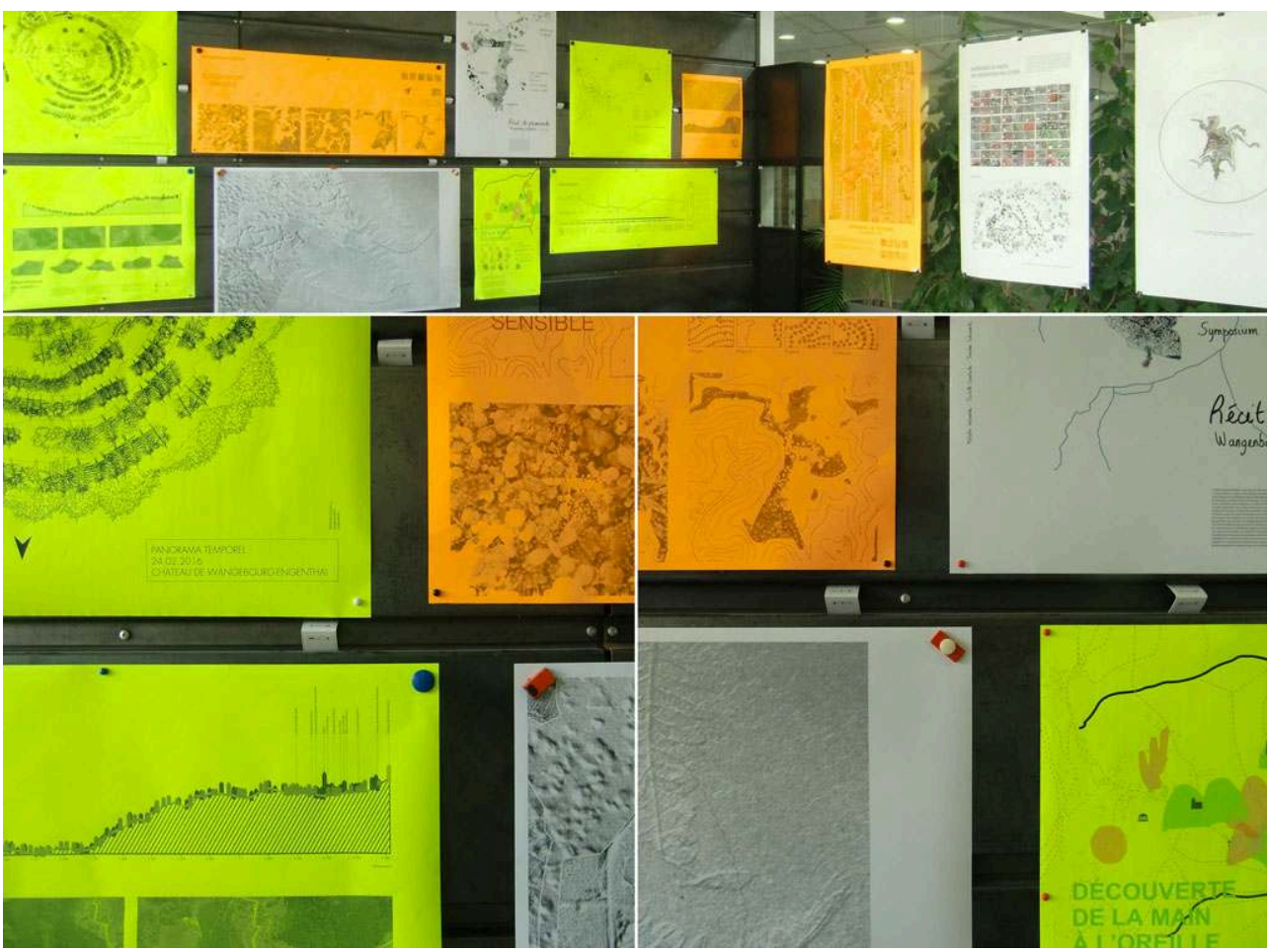

Exposition Cartologies, Strasbourg, 13-24 mars 2016.

Photo : A.-C. Bronner.

\section{Empreintes sensibles}

Parmi les autres travaux, la carte «Empreintes du milieu: une orientation par les sens» (figure 2) se distingue par sa légende comportant 81 entrées distinguant 81 types de surface susceptibles d'être rencontrés aux alentours du château. La création des symboles graphiques se fonde sur deux variables visuelles : la forme et la texture, directement extraits de photographies du terrain, ce qui permet de générer cet ensemble d'idéogrammes illustratifs que les auteurs ont choisi de laisser muets. Au lecteur le choix de nommer le cas échéant les surfaces, qui varient en termes d'échelle, avec des macrophotographies de mousse (tapis étoilé de végétation) ou d'écorce, gros plan d'un regard hydraulique (plaque en fonte) ou de tapis de feuilles, plans d'ensemble des futaies de hêtres et sapins ou de la canopée...

4 La proposition est appliquée à l'échelle d'un plan sommaire du château et de ses environs. L'opacité variable des symboles permet de gérer la densité graphique. Il est nécessaire de recourir à la légende pour identifier le milieu représenté (les différents murs en pierre du château, les tapis de feuilles mortes ou de mousse...). La carte est en cours de construction, tous les espaces ne sont pas renseignés. Si l'échelle semble correcte, le Nord est mal orienté. Un court texte à côté du titre présente l'intention cartographique. 
Figure 2. Empreintes du milieu. Formes et surfaces
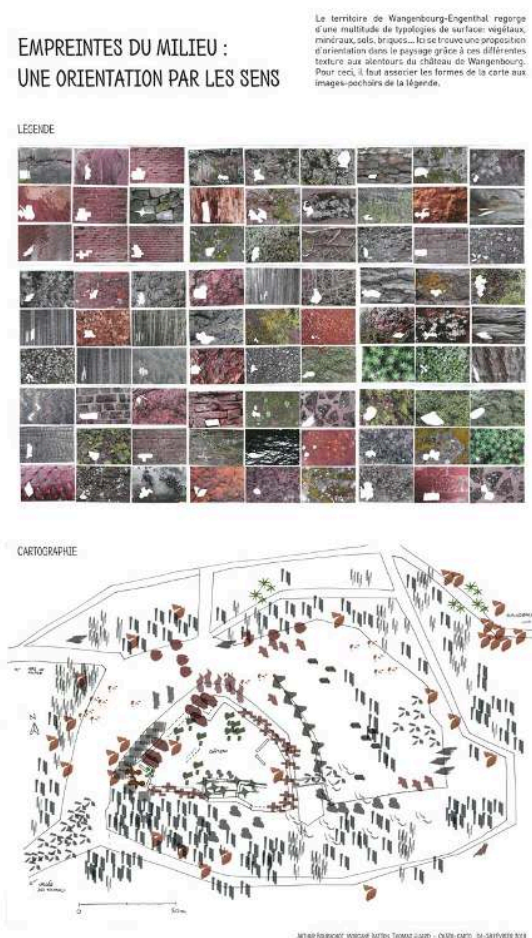

Projet Châto-Carto, exposition Cartologies, Strasbourg, 13-24 mars 2016.

Auteurs : Arthur Bourachot (Master OTG), Morgane Ratton, Thomas Huard (InSituLAB), février 2016.

\section{Le déroulement du workshop}

Le premier jour, sur le terrain, était dédié à la collecte ; le deuxième jour se consacrait à la structuration des informations en vue d'en créer une carte; le troisième jour était centré sur la réalisation et se terminait sur une présentation d'un argumentaire (à l'oral) de la carte réalisée (figure 3). 
Figure 3. Traces du workshop Châto-Carto

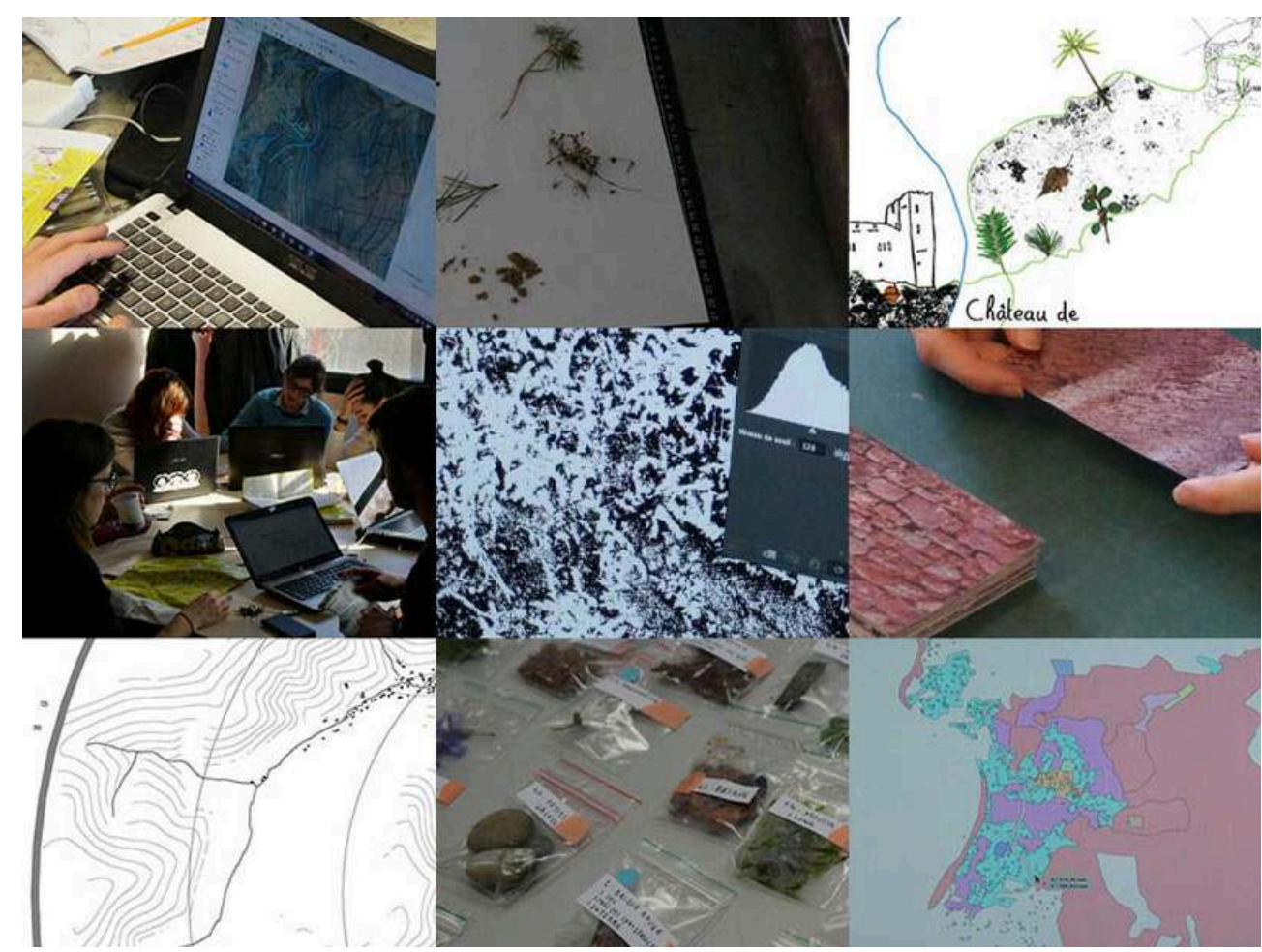

24-26 février 2016.

\section{Jour 1}

6 La collecte des informations sur la commune de Wangenbourg-Engenthal était individuelle. Les étudiants en design sont partis explorer le territoire avec pour consigne: prélever des matériaux, des sons, des couleurs..., réaliser des croquis, photographier des panoramas, interviewer, parcourir le territoire, identifier ce qui est permanent, éphémère, etc. De leur côté, les étudiants géographes ont travaillé sur différents thèmes: l'accessibilité, l'offre en services et équipements, le foncier, la vulnérabilité face au risque, etc., avec des grilles de relevés préalablement construites.

\section{Jour 2}

7 Lors de la seconde journée, à la Faculté de géographie et d'aménagement, l'ensemble des informations, vaste et hétéroclite, a été détaillé, rassemblé, structuré et partagé. Les géographes avaient également mobilisé et présenté différentes couches d'informations spatiales : le bâti, les réseaux, l'hydrographie, le relief, etc., ainsi que les potentialités des outils SIG pour exploiter ces informations spatiales, par exemple calculer des profils topographiques, extraire de zones d'intérêt (périmètre autour d'un parcours...), etc. Des intentions, des thèmes, des pensées ont émergé au sein des douze groupes mixtes formés à partir de cette matière première. 


\section{Jour 3}

Dans les locaux de l'InSituLAB, les intentions cartographiques se sont concrétisées, avec une grande variation tant sur les formes graphiques mobilisées que sur le thème décliné. Dans les faits, les étudiants géographes se sont mis au service des étudiants en design pour intégrer la dimension spatiale dans la production cartographique, comme le souligne la mosaïque figure 4. En fin de journée, cinq minutes ont été accordées à chaque groupe pour présenter leur carte (et échange avec les enseignants présents).

Figure 4. Dimension spatiale dans les cartes sensibles

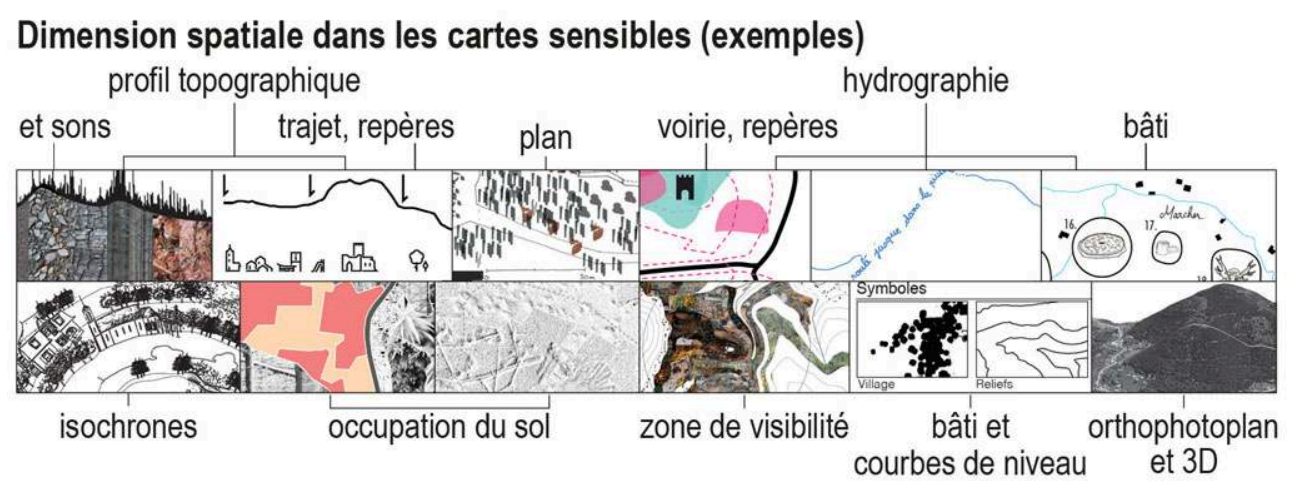

Extraits

\section{Espaces sensibles}

Dans les 12 cartes exposées, l'empreinte de l'espace caractérise cette collaboration entre géographe et designer: isochrones, courbes de niveau ou zone de visibilité, l'expression de coordonnées longitude latitude, de distances métriques ou temporelles à partir d'un lieu, tracé de limites, empreinte des espaces bâtis, tracé des chemins parcourus, localisation des lieux d'attraction, occupation des sols, etc. (figure 4), la " géographie » sert de support pour décliner des thèmes sensibles. Titre et description des œuvres exposées :

- Topographie sonore. Superposition des fréquences sonores enregistrées et du profil topographique d'un parcours, illustrés par des textures graphiques en référence au milieu.

- Randonnée subjective. Parcours et mesures (distance, altitude...) associés à différents pictogrammes et textures.

- Empreintes du milieu. 81 types de milieux d'échelles variables identifiés et cartographiés (figure 2).

- Découverte de la main à l'oreille. Combinaison des tracés de courbes de luminance stylisés, de végétaux ou cailloux prélevés (à toucher) et de sons à écouter, localisés sur un fond de carte.

- Récits de promenades. Invitation à la promenade associant tracés topographiques et textures, éléments végétaux et minéraux, panoramas collectés.

- Le sentier du branquignol. Succession de points d'intérêt, avec variation de la taille des éléments qui se réfèrent au passé ou au présent.

- Panorama temporel. Création de panoramas successifs et concentriques à partir d'un point central. 
- Contrastes et textures. Transformation des aplats colorés standardisés de la carte de l'occupation des sols par des textures en noir et blanc, représentatives de la « réalité du terrain ».

- Texture visuelle. Occupation du sol cartographiée à l'aide de trames construites par un gaufrage à partir d'échantillons prélevés sur le terrain, suggérant les cartes tactiles.

- Perception du paysage depuis le château de Wangenbourg. Carte des "matières" visibles depuis le château, qui peuvent être " palpés » par les habitants et touristes promeneurs.

- Accessibilité sensible. Association d'éléments géographiques (bâti, courbes de niveau...), de matières et de sons.

- Impressions de reliefs. Visions d'un parcours en 1, 2 et 3 dimensions.

10 On regrettera qu'il n'y ait pas eu un intérêt plus marqué de la part des designers aux données mesurées par les géographes ou aux codes de la carte "géographique » (une expression poétique de la luminance d'un objet? Une charte originale pour caractériser la vulnérabilité des bâtis face au risque ?). Ce format très court et intense n'a pas permis d'aller au bout des intentions. Les étudiants des deux disciplines (géomatique/ design) travaillaient, sauf exceptions, en parallèle, et l'absence d'échanges sur les connaissances et les compétences, en amont et en aval, a limité le potentiel de création qui pouvait émerger et le dialogue entre Art et Cartographie. La géomatique et la cartographie sensible ne mobilisent pas les mêmes concepts, méthodes, pratiques. Cependant, ils peuvent dialoguer, pour éveiller a minima la curiosité réciproque, et ne pas cantonner la création en cartographie au champ de l'Art.

Les auteurs des cartes :

InSituLAB : Florent Alexandre, Elin-margot Armannsdottir, Koulma Bilger, Pétronille Camphuis, Sophie Chialva, Solène Dietz, Célia Galipaud, Juliette Ganteille, Tristan Gendre, Thomas Huard, Suzanne Husson, Antoine Jakubczak, Audrey Laurent, Morgane Marin, Pauline Molina, Aurelie Roure, Ibrahim Senol, Agathe Tabeaud, Mélodie Valverde, Morgane Ratton, Mathilde Richard, Jean-Denis Tridon, Maëva Tobalagba, Lena Vercellone.

Master Observation de la Terre et géomatique : Quentin Poterek, Arthur Bourachot, Alexia Cornic, Louis Gangloff, Arnaud Geng, Élodie Ghyselinck, Saber Hajji, Youssouf Chouaibou Marega, Guillaume Matusiak, Xavier Réjaud, Mylene Ritter, Élodie Ruch, Jérôme Schwartz.

\section{NOTES}

1. Colloque Temps Art \& Cartographie - La sémiologie dans tous les sens 16-18 mars 2016. L'exposition Cartologies est née de plusieurs mois de travail portés par Anne-Christine Bronner, cartographe au Laboratoire image, ville, environnement (CNRS-université de Strasbourg), Nicolas Couturier et Bruno Lavelle, enseignants et designers à l'InSituLAB, lycée Le Corbusier, Illkirch-Graffenstaden, et l'implication de leurs collègues pour accompagner et encadrer les différents travaux menés. Lieu : 14-23 mars 2016, MISHA (Maison Interuniversitaire des Sciences de l'Homme-Alsace), Université de Strasbourg. Url : art-carto-semio.sciencesconf.org. 
2. Châtourisme (http://www.lyceelecorbusier.eu/dsaa/?p=1902) est une recherche sur un territoire et ses acteurs : la commune de Wangenbourg-Engenthal, située en Piémont des Vosges du Nord, son château, ses habitants, entre Histoires passées, présentes et à venir. Elle est initiée suite à un partenariat contracté entre le DSAA InSituLAB et l'ADT (Agence du Tourisme du BasRhin).

\section{AUTEUR}

\section{ANNE-CHRISTINE BRONNER}

Laboratoire Sage, CNRS-Université de Strasbourg 